\title{
Rising to the challenge of COVID-19
}

Ursula Hofer, Andrea Du Toit and Ashley York

Scientists around the world have risen to the challenges posed by COVID-19 by rapidly

transforming their laboratories and refocusing their research efforts.

At Nature Reviews Microbiology, we have been inspired by how the scientific community have redirected their research efforts to help tackle the COVID-19 pandemic. This global effort has been instrumental in helping us to understand the epidemiology, dynamics and biological features of the new coronavirus and for paving the way for the development of therapeutics and vaccines. In the first of a new series of Feature articles to highlight these efforts, we interviewed Florian Krammer (professor), Daniel Stadlbauer (postdoctoral fellow) and Fatima Amanat (graduate student) at the Department of Microbiology, Icahn School of Medicine at Mount Sinai, New York, US.

\section{How has your institute adapted to tackling} COVID-19, and what is the impact of this work?

F.K. The Icahn School of Medicine is part of the Mount Sinai Health System, which includes many hospitals in New York. Mount Sinai, on the research side as well as on the hospital side, prepared rapidly for a pandemic as we expected that New York would see cases early on. Although the epidemic started later than expected, it hit the New York metropolitan area very hard. Due to a fantastic collaboration between scientists and clinical staff we managed this crisis very well. We had early nucleic acid testing established, were the first hospital in the nation to have serological assays up and running and were the first to treat patients with convalescent plasma. There was never a sense of chaos, and strong leadership paired with dedication of medical and scientific staff helped us to handle this pandemic very well so far.

We had early nucleic acid testing established, were the first hospital in the [US] to have serological assays up and running and were the first to treat patients with convalescent plasma y an antibody test, which got emergency use authorization from the FDA to detect antibodies binding to the SARS-CoV-2 spike protein. We then transferred our research grade assay to the clinical lab at Mount Sinai to allow screening for convalescent plasma donors. Now this assay is also in use to test both symptomatic and asymptomatic employees at Mount Sinai, and we continue to work closely together with the clinical laboratory.

F.A. As early as January, researchers at Mount Sinai had started working on SARS-CoV-2. Several researchers were studying pathogenesis, establishing animal models to study disease caused by SARS-CoV-2, and making crucial reagents needed to start characterization of immune response in humans post-infection. This work is extremely significant as so much is not known about this novel virus, and several drugs are being investigated as potential therapeutics. In addition, we have developed an antibody test that has received emergency use authorization by the FDA and is being used in clinical settings.

\section{How has your daily work life changed?}

F.K. In mid-January it became clear to me that this coronavirus outbreak first reported in Wuhan, China would likely become a pandemic. For years I have been discussing SARS-CoV in the classroom, highlighting how we escaped a deadly pandemic in 2003 very narrowly. For the last two weeks of January I was in a constant panic that almost paralyzed me, I could not focus on anything else. We had already started to work on reagents for SARS-CoV-2 when the sequence became available in the beginning of January. And to escape this panic I started - together with my team - to work harder, as hard as I could, on reagents and assays for this new virus. Thankfully, we were set up for this because we do similar work with influenza virus. Between the beginning of February and the end of May I probably worked between 13-14hours per day, 7 days a week. Our laboratory developed a serological assay to screen SARS-CoV-2 seroconverters; this was then transferred to the clinical laboratory for screening of plasma donors, and we shared reagents for that assay with more than 250 laboratories worldwide, while maintaining the supply chain of recombinant antigens for our clinical laboratory. In addition, I tried to do as much science outreach and information sharing with the public as possible through Twitter as 


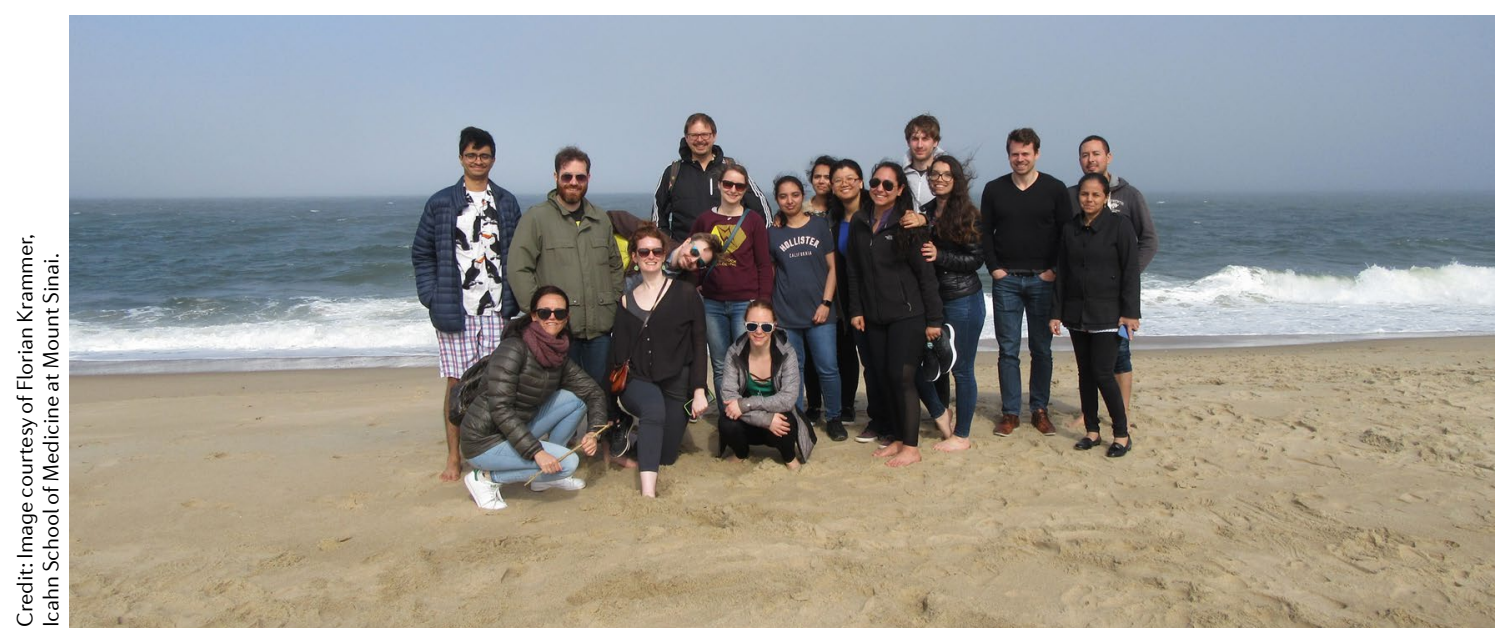

well as traditional media. I work a lot but I have never worked so hard in my whole life as during this time.

D.S. I started to wear a facemask at all times and tried to avoid crowds and other people as much as possible in the work setting (and outside the work setting). Luckily, I live close by to my workplace and can commute by walking, which pretty much avoids the risk of getting exposed on my way to work. At work, my ongoing research projects had to be paused, and I worked hard to transfer and help set up our serological test in the clinical setting. There was an urgent need for such an assay, and it was important to find convalescent plasma donors quickly to have a first tool to treat sick patients available.

F.A. In addition to my $\mathrm{PhD}$ thesis, I immediately started producing recombinant SARS-CoV-2 proteins in February, which were not available anywhere. Next, I started to characterize human immune response and antibody levels in individuals infected with SARS-CoV-2. We were able to develop an antibody test at the beginning of March that could determine if an individual had been exposed to SARS-CoV-2.

\section{What challenges are you facing right now, and what challenges do you anticipate?}

F.K. From March 20 to the end of April, all non-COVID-19 work was on pause at Mount Sinai. The laboratory instead of shutting down like many other laboratories - ramped up. We were working at full capacity on SARS-CoV-2. Then, in the beginning of May, we restarted our influenza virus work that took our full attention pre-COVID-19. The challenge now is to hire additional personnel to keep both streams of work going. We need to continue our work on SARS-CoV-2, but we also need to make progress on influenza virus. The problem is that many of my staff - including myself — are overworked. What we really would need is a long vacation but this seems like something that is very far away.

D.S. New York City is now slowly reopening. It will be interesting to see how well this process works out and if the city will go back to some kind of 'new normal' while we wait for a much needed vaccine. I anticipate that travelling within the US and abroad will still be restricted and not that easy for a while. I am not sure if I will be able to travel back to my home country (and re-enter the US) this year. In the laboratory, I now have to both restart my 'pre-pandemic' research projects and also keep on working on several COVID-19-related projects, which might be challenging.

F.A. Given that SARS-CoV-2 is a novel virus and is highly contagious, it has been hard at times to find resources to perform some experiments as there are not many positive controls available, and so much about the virus is still unknown. In addition, it will be hard to focus on studying other potential emerging viruses as long as the pandemic persists.

What were you working on before the COVID-19 pandemic, and how is this work being impacted?

F.K. Our influenza virus work was only impacted for a relatively short amount of time from the end of March to May. We have now restarted all influenza virus projects, but are struggling with keeping work on both viruses going at full force owing to having too little personnel and being constantly overworked.

D.S. I was doing research on the influenza virus before the pandemic and a lot of influenza virus-related serology. I am interested in antibodies that target the influenza virus neuraminidase and in developing a universal influenza virus vaccine based on hemagglutinin stalk antibodies. This work was on pause for some weeks and is now being resumed.

F.A. Before the pandemic, my PhD thesis work focused on studying the immune response to the glycoproteins of arenaviruses, and this work is aimed at aiding vaccine development. Arenaviruses are highly pathogenic viruses that can cause hemorrhagic fever in humans and have high case fatality rates. This work is being delayed and impacted, because I spend the majority of my time working on SARS-CoV-2; however, I have restarted my projects in the past few weeks.

\footnotetext{
Ursula Hofer $^{\bowtie}$, Andrea Du Toit ${ }^{\bowtie}$ and Ashley York ${ }^{\bowtie}$

Nature Reviews Microbiology.

凶e-mail:nrmicro@nature.com

https://doi.org/10.1038/s41579-020-0419-7
} 